\title{
Comportamiento clínico y gasométrico en falla respiratoria aguda tratada con cánula nasal de alto flujo
}

\author{
Clara Lizeth Palencia-Mojica ${ }^{1 *}$ orcid.org/0000-0002-2649-6572 \\ Adriana Sofía Valero-Ortiz' ${ }^{1}$ orcid.org/0000-0003-3626-8626 \\ Laura Jimena Silva-Rodríguez' ${ }^{1}$ orcid.org/0000-0003-4918-021X
}

Clinical and gasometric behavior in acute respiratory failure treated with a high-flow nasal cannula

1 Grupo de Investigación OXIGENAR, Programa Terapia Respiratoria, Universidad de Boyacá. Tunja, Colombia.

Fecha de aceptación: Abril 30 - 2020

Palencia-Mojica CL, Valero-Ortiz AS, Silva-Rodríguez LJ. Comportamiento clínico y gasométrico en falla respiratoria aguda tratada con cánula nasal de alto flujo. Univ. Salud. 2020;22(2):102-111. DOI: https://doi.org/10.22267/rus.202202.181

\section{Resumen}

Introducción: La cánula nasal de alto flujo en adultos ofrece un tratamiento alternativo a la oxigenoterapia convencional. Objetivo: Establecer el comportamiento clínico y gasométrico de pacientes con falla respiratoria aguda que habitan a 2.600 msnm, tratados con cánula nasal de alto flujo en cuidado intensivo adulto. Materiales y métodos: Estudio piloto cuasi experimental pretest-postest intrasujeto, tamaño de muestra 14 pacientes calculado a través de Epidat. Tipo de muestreo no probabilístico por conveniencia, durante catorce meses. Resultados: El 78\% de los pacientes fueron mujeres, la edad promedio fue 68 años, y el diagnóstico principal fue enfermedad pulmonar obstructiva crónica (EPOC) en el 71,42\% de los casos. Se administró un flujo de oxígeno promedio de $30 \mathrm{Lt} / \mathrm{min}$ para pacientes con (EPOC), para el resto de diagnósticos se programaron flujos mayores. El trabajo respiratorio disminuyó posterior al uso de cánula nasal de alto flujo, la presión arterial de oxígeno mejoró paulatinamente en pacientes con falla hipoxémica y el índice de oxigenación incrementó progresivamente en los dos tipos de falla respiratoria. Conclusiones: La cánula nasal de alto flujo es una opción terapéutica útil en el manejo de la falla respiratoria aguda a 2.600 msnm, permitiendo mejoría de la oxigenación y disminución del trabajo respiratorio.

Palabras clave: Fallo respiratorio; cánula nasal; EPOC; trabajo respiratorio; gasometría (Fuente: DeCS, Bireme).

\begin{abstract}
Introduction: High-flow nasal cannula offers an alternative treatment approach to conventional oxygen therapy in adults. Objective: To establish the clinical and gasometric behavior of patients with acute respiratory failure who live at 2,600 m.a.s.l. and are treated with high-flow nasal cannula in an adult intensive care unit. Materials and methods: Quasi-experimental pilot study with a pretest-posttest within-subject design and a sample size of 14 patients calculated through Epidat. A non-probabilistic convenience sampling was conducted for fourteen months. Results: $78 \%$ of the patients were women and their average age was 68 years. Chronic Obstructive Pulmonary Disease (COPD) was diagnosed in $71.42 \%$ of the cases. An oxygen flow rate of $30 \mathrm{~L} / \mathrm{min}$ was administered to patients with Chronic Obstructive Pulmonary Disease, while higher rates were used for the rest of the diagnoses. Nasal cannula treatment led to (i) a decreased respiratory function, (ii) a gradually improved arterial blood pressure in patients with hypoxemic failure, (iii) and a progressively increased oxygenation rate. Conclusions: High-flow nasal cannula is a useful therapeutic option in the management of patients with acute respiratory failure living at 2,600 m.a.s.l. because it improves oxygenation and reduces respiratory function.
\end{abstract}

Key words: Respiratory failure; nasal cannula; COPD; work of breathing; blood gas analysis (Source: DeCS, Bireme).

\footnotetext{
*Autor de correspondencia

Clara Lizeth Palencia Mojica

e-mail: clpalencia@uniboyaca.edu.co
} 


\section{Introducción}

La falla respiratoria aguda se produce por un aporte insuficiente de oxígeno y eliminación de dióxido de carbono a nivel tisular, lo que representa una incapacidad del sistema respiratorio para suplir las necesidades metabólicas del organismo. Esta insuficiencia puede ser secundaria a problemas en la oxigenación (falla hipoxémica), en la eliminación de dióxido de carbono (falla hipercápnica) o ambas deficiencias de forma simultánea (falla mixta). Para el manejo de este tipo de fallas, el tratamiento va encaminado a mejorar la oxigenación o la ventilación para solucionar la hipoxemia o la hipercapnia. Por este motivo, dentro del manejo de la falla, se encuentra el soporte con oxigenoterapia y una reciente alternativa es el uso de sistemas de alto flujo, que permite mejorar la oxigenación por mecanismos como son la disminución del espacio muerto anatómico, presurización de la vía aérea con cierto grado de presión, disminución de la dilución de oxígeno administrado, aumento del volumen circulante y mejoramiento del transporte mucociliar, entre otras. Diferentes estudios, demuestran que los pacientes con insuficiencia respiratoria mejoran disnea, hipoxemia, manejo de las secreciones y disminución del requerimiento de la ventilación mecánica(1).

El uso de la cánula nasal de alto flujo se ha incrementado en los últimos años, siendo un dispositivo no invasivo, el cual se utilizó inicialmente en unidades de cuidado intensivo neonatal(2), para el manejo de falla respiratoria(3) y procesos de extubación(4), posteriormente se amplió el uso a la población adulta y pediátrica, principalmente para el tratamiento terapéutico de falla hipoxémica(5-7).

En Colombia entre el año 2005 y 2014(8) las principales causas de muerte fueron las enfermedades del sistema circulatorio con un $30,08 \%$, seguidas por un subgrupo de enfermedades entre las cuales se incluyen las enfermedades crónicas de las vías respiratorias inferiores con $20,52 \%$ y las enfermedades transmisibles, que constituyen la primera causa de muerte produciendo el 49,46\% de las defunciones en el país. Frente a las causas de morbilidad en el departamento de Boyacá, las enfermedades no transmisibles ocupan el 64,4\%, siendo las más prevalentes las enfermedades del sistema circulatorio, seguidas de las enfermedades respiratorias las cuales incluyen la enfermedad pulmonar obstructiva crónica, las estadísticas anteriormente mencionadas predisponen la presentación de falla respiratoria aguda, con requerimiento del uso de la cánula de alto flujo(9).

En el contexto internacional existen estudios que permiten evidenciar la efectividad y la utilidad de la cánula nasal de alto flujo (CNAF) en pacientes neonatos y pediátricos con falla respiratoria aguda tipo hipoxémica e hipercápnica(10,11). Frente a la población adulta, los estudios se iniciaron en el año 1994 con la investigación realizado por Dewan y Bell(12), quienes describieron la utilidad de la cánula de alto flujo en adultos con enfermedad pulmonar obstructiva crónica, posteriormente Chatila et al.(13), estudiaron por primera vez el efecto del uso de la cánula de alto flujo en pacientes con enfermedad pulmonar obstructiva crónica, dando como resultado mejor tolerancia al ejercicio y disminución de la disnea. Kernick et al.(14), publicaron la única revisión sistemática en adultos en estado crítico, la cual es limitada teniendo en cuenta que siete de los estudios realizados, se fundamentaron en resúmenes, que no permiten realizar conclusiones solidas que respalden la utilización de la cánula de alto flujo. Posterior a este estudio, se han realizado investigaciones en la aplicación del dispositivo en adultos teniendo en cuenta parámetros clínicos e índices de oxigenación, similar a las variables estudiadas en la presente investigación.

Por las razones anteriormente expuestas, el uso de la cánula nasal de alto flujo, ha cobrado importancia para el manejo de la falla respiratoria, dado que ofrece un tratamiento alternativo de oxigenación gracias al sistema de humidificación, calefacción e interface utilizadas en el mismo, con flujos fijos y presiones dinámicas que mejoran el comportamiento clínico del paciente y procesos fundamentales como la oxigenación y la ventilación. Adicionalmente, los pacientes presentan mayor tolerancia y confort con evidente mejoría de su cuadro clínico(15,16).

De igual manera, los valores de los gases arteriales varían de acuerdo a las condiciones clínicas de los pacientes, y la altura sobre el nivel del mar cobra una importancia fisiológica, porque a medida que se asciende en altura, se presenta una disminución de la presión barométrica, la presión inspirada de oxígeno (PIO2) y la presión arterial de oxígeno (PaO2); estas condiciones de hipoxemia llevan a un aumento de la ventilación minuto $\mathrm{y}$ en consecuencia a la disminución de la presión arterial de dióxido de 
carbono (PaCO2)(17). La relación presión arterial de oxígeno y fracción inspirada de oxígeno (Pa02/FiO2), para altitudes mayores de $1000 \mathrm{msnm}$ según la definición de Berlín, varía dejando el valor de normalidad mayor a $221 \mathrm{mmHg}$, con relación a nivel de mar que es mayor $300 \mathrm{mmHg}(17)$. De acuerdo al último estudio realizado por Lasso en 2014(18), los valores de normalidad de gases arteriales en Bogotá (2.640 msnm) para la PaO2 y la saturación arterial de oxígeno muestran una disminución y con respecto a la presión arterial de dióxido de carbono (PaCO2) aumenta con la edad.

El propósito del estudio fue establecer el comportamiento clínico y gasométrico de los pacientes con falla respiratoria aguda a $2.600 \mathrm{msnm}$, tratados con cánula nasal de alto flujo en la unidad de cuidado intensivo adulto (UCIA) de una institución de tercer nivel del año.

\section{Materiales y métodos}

\section{Diseño y población del estudio}

Estudio piloto cuasi experimental pretest - postest intrasujeto, con un tamaño de muestra de 14 pacientes, calculado a través del paquete estadístico Epidat utilizando la comparación de grupos emparejados. El tipo de muestreo es no probabilístico por conveniencia.

\section{Criterios de inclusión}

Pacientes mayores de 18 años de Unidad de Cuidado Intensivo Adulto que requirieron cánula nasal de alto flujo posterior al uso de soporte ventilatorio y/o de oxigenoterapia, que habitan a $2.600 \mathrm{msnm}$, con diagnóstico de falla respiratoria hipoxémica e hipercápnica y Glasgow mayor de trece.

\section{Criterios de exclusión}

Pacientes con intolerancia al dispositivo administrado y con traqueotomía.

\section{Proceso de recolección de la información}

Los datos se obtuvieron a partir de la revisión de historias clínicas y registro de monitoreo gasométrico y clínico de las variables estudiadas, se recolectaron características demográficas, diagnóstico desencadenante, tipo de falla respiratoria, flujo y fracción inspirada de oxígeno requeridos en cada entidad patológica, soporte ventilatorio y/o de oxigenoterapia utilizado previo y posterior al uso de la cánula, comportamiento clínico y gasométrico de los pacientes a la hora, seis, doce y veinticuatro horas posteriores al uso del dispositivo.

\section{Procesamiento y análisis de la información}

Se realizó a través del paquete estadístico SPSS versión 20. Se realizó análisis univariado para las variables descriptivas teniendo en cuenta las medidas de tendencia central, promedios, desviación estándar e intervalos de confianza y para la fase de correlación la prueba no paramétrica de Friedman.

\section{Consideraciones éticas}

El proyecto estuvo regido por la resolución $N^{\circ} 8430$ del 04 de octubre de 1993(19), esta se clasificó como investigación con riesgo mínimo, se diligenció consentimiento informado por cada uno de los pacientes hospitalizados en la Unidad de Cuidado Intensivo Adulto que participaron en la investigación, antes de instaurar el dispositivo. El proyecto fue aprobado previa revisión y autorización por parte del Comité de Bioética de la Universidad de Boyacá, memorando CB 223 y la institución hospitalaria que participó en el estudio con el código de aprobación número 20162100061291. La información del estudio se manejó con total confidencialidad y privacidad sobre los datos de los pacientes que participaron en el mismo.

\section{Resultados}

\section{Caracterización demográfica}

En la investigación participaron 14 pacientes de los cuales 10 presentaron falla hipercápnica, tres fallas hipoxémicas y uno presentó falla mixta con diagnóstico de osteogénesis imperfecta, patología que afecta la mecánica respiratoria generando en el paciente la presentación de alteración respiratoria restrictiva y obstructiva. Respecto a la variable sexo, el 78\% correspondió al sexo femenino, seguido por el masculino en un $22 \%$, frente a la variable edad los pacientes que requirieron de la cánula nasal de alto flujo tenían edad mínima de 27 y máxima de 91, con edad promedio de 68 años (DE 21,5). La enfermedad pulmonar obstructiva crónica (EPOC) no exacerbada, fue la de mayor presentación con un 71,42\% [IC95\% 47- 94], seguida del tromboembolismo pulmonar (TEP), embolia grasa, neumonía y osteogénesis imperfecta con el 7,14\% respectivamente [IC95\% 120], situación que demuestra que la EPOC es una enfermedad que en el grupo de pacientes estudiados conlleva a la presentación de falla respiratoria hipercápnica caracterizada por hipoventilación alveolar y disminución de la ventilación efectiva. 
El flujo programado en los pacientes con EPOC presentó una mediana de 30 , es decir, que al $50 \%$ de los pacientes se les programó flujo de hasta 30 $\mathrm{Lt} / \mathrm{min}$, flujo que se mantuvo durante las 24 horas de monitoreo; mientras que al $50 \%$ restante, se les programó flujos mayores a $30 \mathrm{Lt} / \mathrm{min}$. En los pacientes con falla respiratoria hipoxémica se inició con flujo máximo de $50 \mathrm{Lt} / \mathrm{min}$ y posteriormente se disminuyó el flujo, finalizando a las 24 horas con flujo de $40 \mathrm{Lt} / \mathrm{min}$ en el $50 \%$ de los monitorizados.

La EPOC presenta una mediana de fracción inspirada de oxígeno (FIO2) en la mayoría de los pacientes del $50 \%$, manteniéndose después de la primera hora de instaurada la cánula nasal de alto flujo, la FIO2 mínima en estos pacientes fue de $30 \%$ y la máxima $68 \%$. Frente a la falla respiratoria hipoxémica la mediana de la FIO2 fue de 40\%, con mínima de 38 y máxima de $60 \%$.

El soporte ventilatorio y/o de oxigenoterapia previo a la instauración de la cánula nasal de alto flujo correspondió en un $71 \%$ a la ventilación mecánica no invasiva, seguido del soporte ventilatorio invasivo con un 21\%. El soporte ventilatorio y de oxigenoterapia posterior al uso de la cánula de alto flujo, en una mayor proporción correspondió al sistema de bajo flujo (cánula nasal convencional) en un $86 \%$, con un flujo promedio de $3 \mathrm{Lt} / \mathrm{min}$.

Frente al comportamiento clínico, se observó que previo a la instauración del dispositivo, la mayoría de los pacientes mostraron algunos signos de dificultad respiratoria como desbalance toracoabdominal, uso de musculatura accesoria y aumento de frecuencia respiratoria (polipnea), lo que permitió evidenciar presencia de trabajo respiratorio, el cual disminuyó de manera significativa desde la primera hora de uso de la cánula, al igual que se observó que con el uso del dispositivo no se generaba presencia de broncoespasmo (Figura 1).

\section{Comportamiento clínico y gasométrico}

En la tabla 1, se observa que, en los diez pacientes con diagnóstico de EPOC, con mediana de administración de $\mathrm{FiO} 2$ de 50\%, la frecuencia respiratoria disminuyo a lo largo del uso de la cánula nasal de alto flujo. Frente al porcentaje de saturación de oxígeno y teniendo en cuenta la altura sobre el nivel del mar a la cual están sometidos los pacientes observados, se evidenció un comportamiento homogéneo y dentro de los valores esperados para el tipo de paciente.

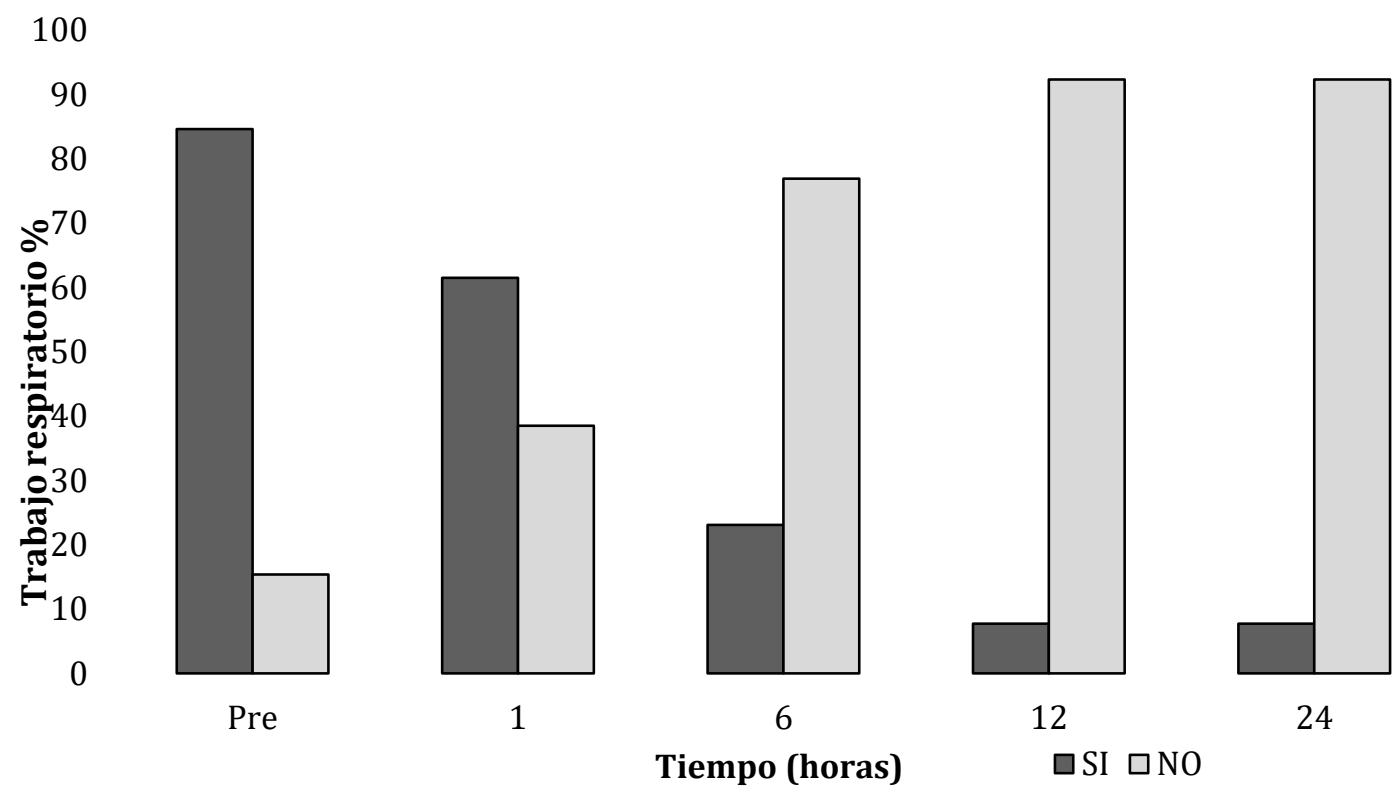

Figura 1. Resultados porcentuales del trabajo respiratorio durante las 24 horas de uso de la cánula nasal de alto flujo, en pacientes con falla respiratoria aguda tratados en la Unidad de Cuidado Intensivo Adulto, en la ciudad de Tunja. 
De acuerdo a datos gasométricos se evidencia que respecto a los valores de normalidad a la altura (2.600 msnm) se presenta $\mathrm{pH}$ previo y posterior al uso de la cánula nasal de alto flujo dentro de los valores de referencia según los estudios de gases. En relación a la $\mathrm{PaO} 2$ el valor previo es más bajo en relación al valor de normalidad para la altura, que es de $60 \mathrm{mmHg}$ y con la PaCO2 su valor también se encuentra elevado con respecto al valor de normalidad para la altura, asociándose además a lo esperado para las fallas respiratorias.

Los parámetros gasométricos evaluados a través de comparación de medidas repetitivas y suma de rangos promedios, las variables de $\mathrm{pH}, \mathrm{PaO} 2 \mathrm{y}$ $\mathrm{PaCO} 2$ se mantuvieron homogéneas sin rechazo de la hipótesis nula, ni diferencias en las mediciones realizadas. Respecto al índice de oxigenación (PaO2/FiO2) se evidenciaron incrementos progresivos con el uso del dispositivo.

De los tres pacientes con falla hipoxémica con diagnóstico de TEP, Embolia Grasa y Neumonía, la mediana de FIO2 fue de 40\%. Frente a la comparación de medidas repetitivas y suma de rangos promedios, las variables $\mathrm{pH}$ y $\mathrm{PaCO} 2$ se mantuvieron homogéneas sin rechazo de la hipótesis nula, respecto a la $\mathrm{PaO} 2$ y al índice de oxigenación, la mediana presenta un incremento gradual con el uso del dispositivo (Tabla 2).

En la figura 2 se observa que en la falla hipercápnica no se presentó aumento de la presión arterial de dióxido de carbono (PaCO2), durante las 24 horas de uso de la cánula de alto flujo. En los pacientes con falla hipoxémica se evidencia una disminución de este parámetro a las 24 horas.

En la figura 3 se evidencia que la presión arterial de oxígeno se mantuvo con una media de $56 \mathrm{mmHg}$ tanto en la etapa previa como en la post administración de la cánula en los pacientes con falla hipercápnica; en la falla hipoxémica la media de la presión arterial de oxígeno ( $\mathrm{PaO} 2)$ se incrementó y mantuvo sobre los $60 \mathrm{mmHg}$ a partir de las 6 horas de uso de la cánula, valor acorde a los metros sobre el nivel del mar y a la presión barométrica de la ciudad donde fueron estudiados los pacientes.

Tabla 1. Parámetros clínicos y gasométricos de los pacientes con falla hipercápnica antes y después del uso de la cánula de alto flujo

\begin{tabular}{|c|c|c|c|c|c|c|c|c|c|c|c|c|c|c|c|c|}
\hline \multicolumn{17}{|c|}{ Falla Respiratoria Hipercápnica } \\
\hline & \multicolumn{3}{|c|}{ Pre } & \multicolumn{3}{|c|}{1 hora } & \multicolumn{3}{|c|}{6 horas } & \multicolumn{3}{|c|}{12 horas } & \multicolumn{3}{|c|}{24 horas } & \multirow[b]{2}{*}{$\mathbf{p}$} \\
\hline & Me & $\mathbf{P}_{25}$ & $\mathbf{P}_{75}$ & Me & $\mathbf{P}_{25}$ & $\mathbf{P}_{75}$ & Me & $\mathbf{P}_{25}$ & $\mathbf{P}_{75}$ & Me & $\mathbf{P}_{25}$ & $\mathbf{P}_{75}$ & Me & $\mathbf{P}_{25}$ & $\mathbf{P}_{75}$ & \\
\hline FR & 22,5 & 20,0 & 24,0 & 22,0 & 20,0 & 27,0 & 22,5 & 20,0 & 25,0 & 21,0 & 19,0 & 23,0 & 20,0 & 19,0 & 22,0 & 0,062 \\
\hline PH & 7,37 & 7,29 & 7,44 & 7,37 & 7,28 & 7,45 & 7,42 & 7,30 & 7,47 & 7,40 & 7,33 & 7,45 & 7,39 & 7,38 & 7,44 & 0,369 \\
\hline $\mathrm{PaO}_{2}$ & 57,5 & 50,0 & 64,0 & 55,0 & 47,0 & 60,0 & 59,0 & 47,0 & 60,0 & 56,5 & 54,0 & 60,0 & 56,0 & 54,0 & 59,0 & 0,199 \\
\hline $\mathrm{PaCO}_{2}$ & 48,0 & 44,0 & 55,0 & 48,0 & 44,0 & 55,0 & 47,0 & 42,0 & 52,0 & 46,0 & 45,0 & 53,0 & 46,0 & 40,0 & 56,0 & 0,225 \\
\hline $\mathrm{PaO}_{2} / \mathrm{FiO}_{2}$ & 113 & 102 & 182 & 112 & 104 & 135 & 118 & 103 & 135 & 127 & 109 & 145 & 126 & 108 & 142 & 0,297 \\
\hline $\mathrm{SpO}_{2}$ & 87,5 & 84,0 & 90,0 & 86,5 & 83,0 & 90,0 & 87,0 & 84,0 & 91,0 & 87,5 & 86,0 & 88,0 & 86,5 & 86,0 & 90,0 & 0,190 \\
\hline
\end{tabular}

FR: frecuencia respiratoria, PH: potencial de hidrogeno, Pa02: presión arterial de oxígeno, PaCO2: presión arterial de dióxido de carbono, PaO2/FiO2: índice de Kirby, SpO2: saturación porcentual de oxígeno.

Tabla 2. Parámetros clínicos y gasométricos de los pacientes con falla hipoxémica antes y después del uso de la cánula de alto flujo

\begin{tabular}{|c|c|c|c|c|c|c|c|c|c|c|c|c|c|c|c|c|}
\hline \multicolumn{17}{|c|}{ Falla Respiratoria Hipoxémica } \\
\hline & \multicolumn{3}{|c|}{ Pre } & \multicolumn{3}{|c|}{1 hora } & \multicolumn{3}{|c|}{6 horas } & \multicolumn{3}{|c|}{12 horas } & \multicolumn{3}{|c|}{24 horas } & \multirow[b]{2}{*}{$\mathbf{p}$} \\
\hline & Me & P25 & P75 & Me & P25 & P75 & Me & P25 & P75 & Me & P25 & P75 & Me & P25 & P75 & \\
\hline FR & 22,0 & 20,0 & 27,0 & 20,0 & 20,0 & 26,0 & 20,0 & 18,0 & 23,0 & 19,0 & 18,0 & 21,0 & 18,0 & 17,0 & 18,0 & 0,028 \\
\hline PH & 7,40 & 7,38 & 7,45 & 7,42 & 7,39 & 7,46 & 7,44 & 7,39 & 7,45 & 7,45 & 7,40 & 7,45 & 7,45 & 7,43 & 7,50 & 0,208 \\
\hline PaO2 & 56,0 & 48,0 & 72,0 & 58,0 & 54,0 & 78,0 & 60,0 & 55,0 & 80,0 & 60,0 & 56,0 & 70,0 & 62,0 & 59,0 & 69,0 & 0,444 \\
\hline $\mathrm{PaCO} 2$ & 35,0 & 27,0 & 37,0 & 35,0 & 29,0 & 36,0 & 35,0 & 29,0 & 37,0 & 35,0 & 29,0 & 38,0 & 33,0 & 28,0 & 35,0 & 0.385 \\
\hline Pa02/FiO2 & 160 & 120 & 180 & 165 & 120 & 195 & 171 & 122 & 205 & 171 & 124 & 184 & 177 & 131 & 209 & 0,035 \\
\hline Sp02 & 88,0 & 85,0 & 93,0 & 89,0 & 88,0 & 96,0 & 90,0 & 87,0 & 98,0 & 91,0 & 89,0 & 96,0 & 94,0 & 92,0 & 94,0 & 0,130 \\
\hline
\end{tabular}

FR: frecuencia respiratoria, PH: potencial de hidrogeno, PaO2: presión arterial de oxígeno, PaCO2: presión arterial de dióxido de carbono, Pa02/FiO2: índice de Kirby, Sp02: saturación porcentual de oxígeno. 


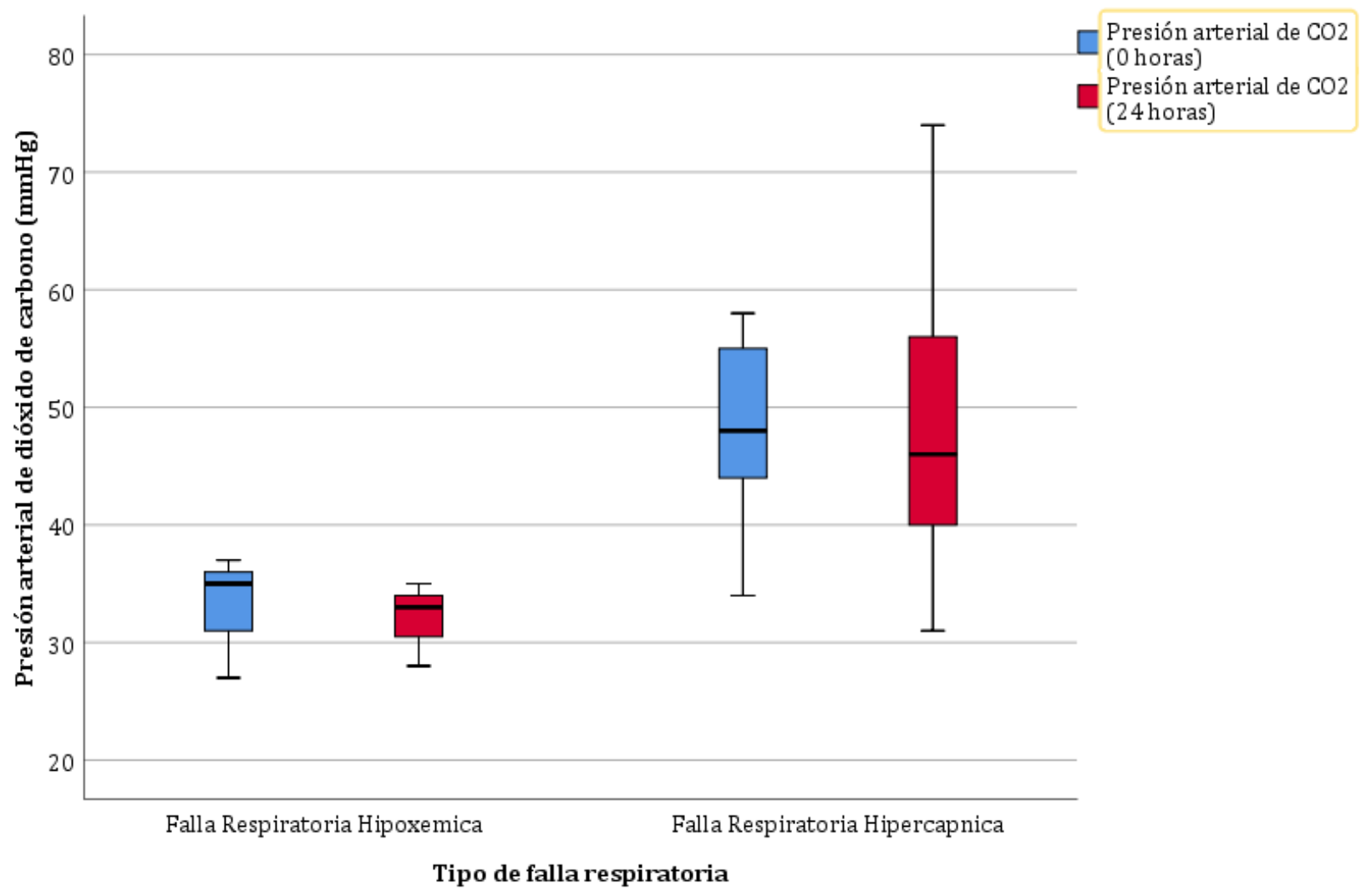

Figura 2. Relación entre el tipo de falla respiratoria y la presión arterial de dióxido de carbono al inicio y al finalizar la terapia con cánula nasal de alto flujo.

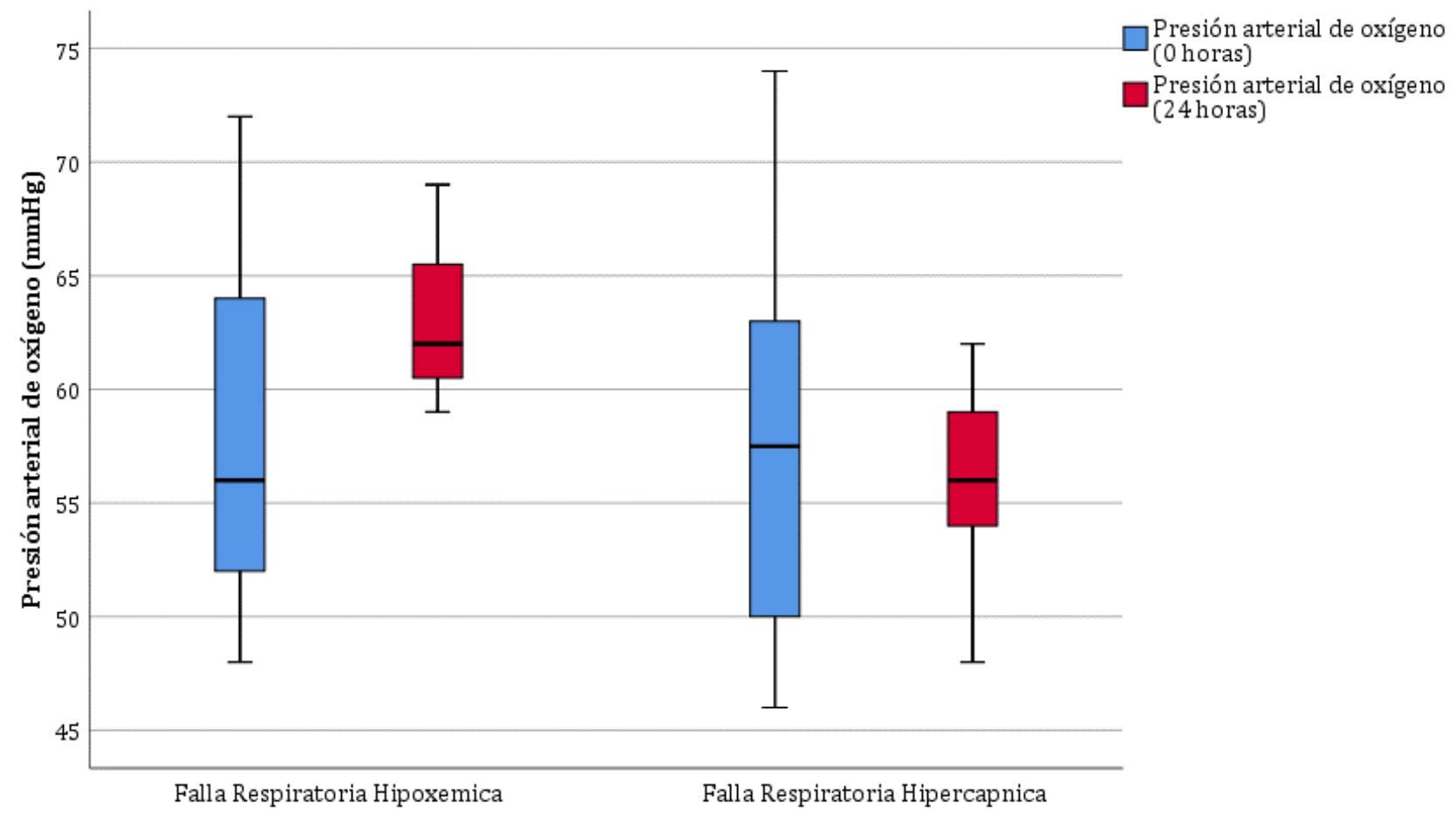

Tipo de falla respiratoria

Figura 3. Relación entre el tipo de falla respiratoria y la presión arterial de oxígeno al inicio y al finalizar la terapia con cánula nasal de alto flujo. 
Respecto al paciente con diagnóstico de osteogénesis imperfecta, el comportamiento con el uso de la cánula nasal de alto flujo corresponde a falla respiratoria mixta, presentando valores de hipercapnia persistente e hipoxemia, dadas las condiciones fisiopatológicas propias de esta entidad. Sin embargo, se evidencio disminución del trabajo respiratorio dado por mejoría en los signos de dificultad respiratoria (uso de musculatura accesoria y polipnea), durante el uso del dispositivo.

\section{Discusión}

La cánula de alto flujo es un sistema de oxigenoterapia que ofrece flujos altos de oxígeno entre 20 - $60 \mathrm{lpm}$, en condiciones casi fisiológicas de calor y humedad, además este dispositivo garantiza una fracción inspirada de oxígeno constante y eficaz, disminuye el espacio muerto, mejora el reclutamiento alveolar $\mathrm{y}$ el gas calentado $\mathrm{y}$ humidificado, además mejora la tolerancia y confort del paciente(20,21). El uso del dispositivo en las unidades de cuidado intensivo adulto se ha incrementado en los últimos años y principalmente en el manejo de la falla respiratoria hipoxémica, en menor proporción en falla respiratoria hipercápnica(22), presentando mejoría clínica al ser tratados con la cánula de alto flujo. Los estudios evidencian que el uso de este dispositivo en falla respiratoria hipercápnica estable, puede mejorar variables clínicas como el patrón respiratorio, el trabajo respiratorio y los niveles de presión arterial de dióxido de carbono, sin embargo, el uso de la cánula nasal de alto flujo, tiene limitaciones en exacerbaciones graves ${ }^{(23,24) \text {. }}$

En el contexto nacional existen pocos estudios que permitan evidenciar los beneficios que la cánula nasal de alto flujo genera en los pacientes adultos con falla respiratoria aguda a grandes alturas, los estudios más recientes se han sido realizado en población pediátrica y neonatal, como el descrito por Vásquez-Hoyos et al.(25) en la cual se concluye que la cánula nasal de alto flujo ha evitado complicaciones y tiene bajas tasas de fracasos, al igual que el estudio referido por el mismo autor(26) en población neonatal, donde se evidenció que la cánula nasal de alto flujo disminuyó la necesidad de soportes respiratorios más invasivos, ambos estudios realizados a grandes alturas. De esta manera, se evidencia que el uso del dispositivo a grandes alturas, genera efectos positivos sobre el comportamiento clínico de los pacientes.

Teniendo en cuenta las variables sociodemográficas descritas en el estudio de Parke et al.(27), se evidenció que la edad media fue de 31 años en hombres, diferente a la población del presente estudio donde la edad promedio fue de 68 años con mayor presentación en mujeres. En el estudio de Bräunlich(28) se observa que la edad promedio de los pacientes a los cuales se les administró la terapia de alto flujo fue de 67,7 años situación similar, a la edad promedio de los pacientes del presente estudio.

Según el estudio realizado por Demelo-Rodríguez et al.(29), la muestra fue de tres pacientes que desarrollaron falla respiratoria aguda en los cuales se reportó edad promedio de 76 años y diagnósticos que conllevaron a falla respiratoria hipoxémica, utilizando flujo de 50 Lts $/ \mathrm{min}$ similares a los utilizados en los pacientes del presente estudio, denotando que los cambios gasométricos y clínicos obtenidos reflejan una similitud con los datos de los pacientes analizados, las variables como $\mathrm{pH}$ se mantuvieron homogéneas y dentro de rangos de normalidad durante el tratamiento. Frente al índice de oxigenación se evidenció mejoría progresiva en las 24 horas alcanzando índices por encima de 120 y con un PaCO2 que para la investigación de Demelo, aunque tiene valores menores a los referidos en el estudio no varían en las 24 horas estudiadas, al igual que la presente investigación. Para el comportamiento clínico la frecuencia respiratoria disminuyó en todos los casos, similar a lo reportado anteriormente.

Dentro de las indicaciones para el requerimiento del uso de la CNAF se encuentra la falla respiratoria hipoxémica, como lo señala Sztrymf y Messika en su estudio(30), una diferencia frente a la utilización de la cánula de alto flujo en el presente estudio es la inclusión de pacientes con insuficiencia respiratoria hipercápnica que presentaron leve trabajo respiratorio asociado a EPOC no exacerbado y donde es evidente la mejoría clínica de los mismos una vez tratados con el dispositivo.

En el estudio realizado por Fraser JF et al.(31), se describe el uso de este tipo de cánula en pacientes críticos, incluyendo 30 casos de pacientes crónicos, analizando variables similares a las del presente estudio, estos autores evidenciaron disminución del 
trabajo respiratorio a través de la frecuencia respiratoria y la disnea.

Antes de instaurar la cánula nasal de alto flujo, el $71 \%$ de los sujetos recibieron presión positiva dada por ventilación mecánica no invasiva y el $86 \%$ posterior al uso del dispositivo de alto flujo, requirieron de cánula nasal de bajo flujo sin requerimiento de ventilación mecánica invasiva. Datos similares se reportan por Gaunt et al.(32), donde estudiaron la falla respiratoria hipoxémica evidenciando que el $14,5 \%$ de la población recibieron ventilación mecánica posterior al uso de CNAF y un $61,3 \%$ no requirieron de soporte ventilatorio posterior al uso de la CNAF. Al igual que en el estudio de Nagata et al.(33) donde posterior al uso de la CNAF menos sujetos requirieron ventilación mecánica no invasiva e invasiva.

En cuanto al comportamiento clínico los resultados del presente estudio evidencian que el trabajo respiratorio disminuye posterior a la instauración de la $\mathrm{CNAF}$, al igual que la frecuencia respiratoria, como lo reporta el estudio de Rittayamai et al.(34), encontrando como desencadenante del trabajo respiratorio patologías como la EPOC y la neumonía. Posterior al uso de la CNAF mejoró significativamente la disnea comparado con la oxigenoterapia de bajo flujo. En el estudio realizado por Demelo Rodríguez, $\mathrm{P}$ y Olmedo Samperio et al.(35), se evaluó la eficacia y tolerancia a la oxigenoterapia con cánula nasal de alto flujo en una unidad de cuidados intermedios, con patologías como enfermedad pulmonar obstructiva crónica, insuficiencia cardíaca, asma e infección respiratoria, mostrando como resultados adecuada tolerancia al dispositivo y evolución clínica favorable al igual que en el estudio presentado y que a pesar del pequeño tamaño muestral es una alternativa de manejo útil en la falla respiratoria como soporte respiratorio.

Lo anteriormente descrito, es similar a estudios realizados en paciente con falla respiratoria hipercápnica(36), donde se inicia con flujos de 35 Lt/min, con aumento del litraje a tolerancia del paciente, para la presente investigación se usaron flujos de 25 a $45 \mathrm{Lt} / \mathrm{min}$ en pacientes con EPOC. Para el caso de falla respiratoria hipoxémica, según las investigaciones los flujos de inicio son de 50 a 60 $\mathrm{Lt} / \min (37)$, alcanzado en el presente trabajo flujos de 25 a $50 \mathrm{Lt} / \mathrm{min}$.
En el presente estudio se demostró que la cánula de alto flujo es un soporte de oxigenoterapia útil en falla respiratoria hipoxémica e hipercápnica y en esta última se evidencia que la presión arterial de dióxido de carbono no aumenta. Además, frente al comportamiento clínico se observa mejoría del trabajo respiratorio, con diminución de signos de dificultad respiratoria como desbalance toracoabdominal, uso de musculatura accesoria y aumento de frecuencia respiratoria (polipnea). Aunque sería útil realizar un estudio con un número más amplio de pacientes en quienes se pueda determinar la eficacia de uso de este dispositivo en falla hipercápnica.

\section{Conclusiones}

El uso de la cánula nasal de alto flujo es una estrategia útil a gran altura en el manejo de la falla respiratoria hipoxémica e hipercápnica, permitiendo una mejoría de la oxigenación y disminución del trabajo respiratorio, además de ser una alternativa de tratamiento de la ventilación mecánica. Sin embargo, se necesita contar con estudios multicéntricos con una muestra más representativa de pacientes que permitan establecer la eficacia de la cánula de alto flujo en las diferentes fallas respiratorias.

\section{Limitaciones del estudio}

La principal limitación del estudio fue el tamaño de la muestra. Sigue siendo importante contar con estudios de mayor muestra que avalen la eficacia y seguridad de esta terapia en los pacientes con falla respiratoria aguda.

\section{Agradecimientos}

A la Universidad de Boyacá por la financiación de la investigación y a la Institución Hospitalaria por su apoyo para la realización del estudio.

Conflicto de intereses: Los autores declaran no tener ningún conflicto de interés.

\section{Referencias}

1. Dueñas Castell C, Mejía Bermúdez J, Coronel C, Ortiz Ruíz G. Insuficiencia respiratoria aguda. Acta Colomb Cuid Intensivo. 2016;16(S1):1-24. http://doi.org/10.1016/j.acci.2016.05.001.

2. Masclans JR, Pérez Terán P, Roca 0 . The role of high-flow oxygen therapy in acute respiratory failure. Medicina Intensiva. https://doi.org/10.1016/j.medin.2015.05.009. 
3. Mauri T, Turrini C, Eronia N, Grasselli G, Volta CA, Bellani G, Pesenti A. Physiologic effects of high-flow nasal cannula in acute hypoxemic respiratory failure. Am J Respir Crit Care Med. 2017;195(9):1207-1215. https://doi.org/10.1164/rccm.201605-09160C.

4. Song HZ, Gu JX, Xiu HQ, Cui W, Zhang GS. The value of highflow nasal cannula oxygen therapy after extubation in patients with acute respiratory failure. Clinics (Sao Paulo). 2017;72(9):562-567.

https://doi.org/10.6061/clinics/2017(09)07.

5. Lee JH, Rehder KJ, Williford L, Cheifetz IM, Turner DA. Use of high flow nasal cannula in critically ill infants, children, and adults: a critical review of the literature. Intensive Care Med. 2013;39(2): 247-257. doi: 10.1007/s00134-012-2743-5.

6. Delorme M, Bouchard PA, Simon M, Simard M, Lellouche F. Effects of High-Flow Nasal Cannula on the Work of Breathing in Patients Recovering From Acute Respiratory Failure. Crit Care Med. 2017;45(12):1981-1988. https://doi.org/10.1097/CCM.0000000000002693.

7. Frat JP, Thille AW, Mercat A, Girault C, Ragot S, Perbet S, et al. High-flow oxygen through nasal cannula in acute hypoxemic respiratory failure. $\mathrm{N}$ Engl J Med. 2015;372:2185-2196.

https://doi.org/10.1056/NEJMoa1503326.

8. COLOMBIA. MINISTERIO DE SALUD Y PROTECCION SOCIAL. Análisis de situación de salud (ASIS) Colombia, 2016. [En línea]. Bogotá: El Ministerio, 2016. [Citado el 13-09-2017]. Disponible en: https://www.minsalud.gov.co/sites/rid/Lists/BibliotecaDi gital/RIDE/VS/ED/PSP/asis-colombia2016.pdf.

9. BOYACA. GOBERNACION. SECRETARIA DE SALUD. Análisis de situación de salud con el modelo de los determinantes sociales de salud, Boyacá, 2016. [En línea]. Tunja: La Gobernación, 2016. [Citado el 13-09-2017]. Disponible en https://www.boyaca.gov.co/SecSalud/images/Documentos /asis2016/asis_departamental_2016.pdf.

10. Nishimura M. High-Flow Nasal Cannula Oxygen Therapy in Adults: PHysiological Benefits, Indication, Clinical Benefits, and Adverse Effects. Respir Care. 2016;61(4):529-541. doi: 10.4187 /respcare.04577.

11. Ito J, Nagata $K$, Sato $S$, Shiraki A, Nishimura N, Izumi S, Tachikawa R, Morimoto T, Tomii K. The clinical practice of high-flow nasal cannula oxygen therapy in adults: A Japanese cross-sectional multicenter survey. Respir Investig. $2018 \quad$ May;56(3):249-257. doi: 10.1016/j.resinv.2018.02.002. Epub 2018 Mar 10.

12. Dewan NA, Bell CW. Effect of low flow and high flow oxygen deliveryon exercise tolerance and sensation of dyspnea. A study comparing the transtracheal catheter and nasal prongs. Chest. 1994 Apr;105(4):1061-1065. doi: 10.1378/chest.105.4.1061.

13. Chatila W, Nugent $T$, Vance G, Gaughan J, Criner GJ. The effects of high-flow vs low-flow oxygen on exercise in advanced obstructive airways disease. Chest. 2004 Oct;126(4):1108-1115. doi: 10.1378/chest.126.4.

14. Kernick J, Magarey J. What is the evidence for the use of high flow nasal cannula oxygen in adult patients admitted to critical care units? A systematic review. Aust Crit Care. 2010 May;23(2):53-70. doi: 10.1016/j.aucc.2010.01.001.

15. Vargas F, Saint-Leger M, Boyer A, Bui NH, Hilbert G. Physiologic effects of high-flow nasal cannula oxygen in critical care subjects. Respir Care. 2015;60(10):1369-1376. doi: $10.4187 /$ respcare.03814.
16. Segovia B, Velasco D, Jaureguizar Oriol A, Díaz Lobato S. Combination Therapy in Patients with Acute Respiratory Failure: High-Flow Nasal Cannula and Non-Invasive Mechanical Ventilation. Arch. Bronconeumol. 2019 Mar;55(3):166-167. doi: 10.1016/j.arbres.2018.06.002.

17. Patiño JF. Gases sanguíneos, fisiología de la respiración e insuficiencia respiratoria aguda. 8th. Ed. Bogotá: Editorial Médica Internacional; 2015.

18. Lasso Apráez JI. Interpretación de los gases arteriales en Bogotá (2.640 msnm) basada en el nomograma de SiggaardAndersen. Una propuesta para facilitar y unificar la lectura. Rev Colomb Neumol. 2014;26(1):25-36. Disponible en: https://revistas.asoneumocito.org/index.php/rcneumologi a/article/view/56.

19. MINISTERIO DE SALUD RESOLUCION NUMERO 8430 DE 1993 [En línea]. Bogotá: Ministerio de Salud, 2016. [Citado el 13-05-2018]. Disponible en: https://www.minsalud.gov.co/sites/rid/Lists/BibliotecaDi gital/RIDE/DE/DIJ/RESOLUCION-8430-DE-1993.pdf.

20. Spoletini G, Alotaibi M, Blasi F, et al. Heated humidified highflow nasal oxygen in adults: mechanisms of action and clinical implications. Chest. 2015;148:253-61. doi: 10.1378/chest.14-2871.

21. Cuquemelle E, Pham T, Papon JF, Louis B, Danin PE, Brochard L. Heated and humidified high-flow oxygen therapy reduces discomfort during hypoxemic respiratory failure. Respir Care. 2012;57(10):1571-1577. https://doi.org/10.4187/respcare.01681.

22. Di Mussi R, Spadaro S, Stripoli T, Volta CA, Trerotoli P, Pierucci $\mathrm{P}$, et al. High-flow nasal cannula oxygen therapy decreases postextubation neuroventilatory drive and work of breathing in patients with chronic obstructive pulmonary disease. Crit Care. 2018 Aug 2;22(1):180. doi: 10.1186/s13054-018-2107-9.

23. Bräunlich J, Köhler M, Wirtz H. Nasal high-flow improves ventilation in patients with COPD. IJCOPD. 2016;11(1):1077-1085. https://doi.org/10.2147/COPD.S104616.

24. Mortaz E, Jamaati H. The First Symposium on COPD and Therapeutic Approaches 2016, Tehran, Iran. Tanaffos [Internet]. 2017;16(Suppl 1):S1-S1. Available from: https://pubmed.ncbi.nlm.nih.gov/29158742.

25. Vásquez-Hoyos $P$, Jiménez Chaves $A$, Tovar $M$, Albor $R$, Palencia M, Redondo D, Roa J. Factores asociados al fracaso de la terapia con cánulas nasales de alto flujo en pacientes pediátricos con insuficiencia respiratoria en dos unidades de cuidados críticos pediátricos a gran altitud. Medicina Intensiva.

2019. https://doi.org/10.1016/j.medin.2019.10.005.

26. Vásquez-Hoyos $\mathrm{P}$, Arias-Fernández DA, Barrios-Marenco A, Álvarez-Fonseca YC, Soler-Rincón PJ. Experiencia del uso de la cánula nasal de alto flujo en cuidados intensivos neonatales de un hospital a 2,600 metros sobre el nivel del mar. Rev Mex Pediatr. 2018;85(2):60-65. Disponible en: https://www.medigraphic.com/pdfs/pediat/sp2018/sp182d.pdf.

27. Parke RL, Bloch A, McGuinness SP. Effect of very-high-flow nasal therapy on airway pressure and end-expiratory lung impedance in healthy volunteers. Respir Care. 2015;60(10):1397-403. doi: 10.4187/respcare.04028.

28. Bräunlich J, Beyer D, Mai D, Hammerschmidt S, Seyfarth H-J, Wirtz H. Effects of Nasal High Flow on Ventilation in Volunteers, COPD and Idiopathic Pulmonary Fibrosis 
Patients. Respiration. 2013;85:319-325. doi: 10.1159/000342027.

29. Demelo P, Olmedo M, Gaitán DG, Cano JC, Andueza Lillo JA. Oxigenoterapia de alto flujo con cánula nasal: estudio preliminar en pacientes hospitalizados. Arch Bronconeumol. 2015;51(12):657-659. doi: 10.1016/j.arbres.2015.03.015.

30. Sztrymf B, Messika J, Mayot T, Lenglet H, Dreyfuss D, Richard JD. Impact of high - flow nasal cannula oxygen therapy on intensive care unit patients with acute respiratory failure: a prospective observational study. J Crit Care. 2012;27(3):324e9-13. doi: 10.1016/j.jcrc.2011.07.075.

31. Fraser JF, Spooner AJ, Dunster KR, et al. Nasal high flow oxygen therapy in patients with COPD reduces respiratory rate and tissue carbon dioxide while increasing tidal and end-expiratory lung volumes: a randomised crossover trial. Thorax. 2016;71(8):759-761. doi: 10.1136/thoraxjnl-2015207962.

32. Gaunt K, Spilman S, Halub M, Jackson J, Lamb K, Sahr S. High-Flow Nasal Cannula in a Mixed Adult ICU. Respir Care. 2015;60(10):1383-1389. doi: 10.4187/respcare.04016.

33. Nagata K, Morimoto T, Fujimoto D, Otoshi T, Nakagawa A, Otsuka K, et al. Efficacy of High-Flow Nasal Cannula Therapy in Acute Hypoxemic Respiratory Failure: Decreased Use of Mechanical Ventilation. Respir Care. 2015;60(10):1390-6. doi: $10.4187 /$ respcare.04026.

34. Rittayamai N, Tscheikuna J, PrapHruetkit N, Kijpinyochai S. Use of High-Flow Nasal Cannula for Acute Dyspnea and Hypoxemia in the Emergency Department. Respir Care. 2015;60(10):1377-82. doi: 10.4187/respcare.03837.

35. Demelo $\mathrm{P}$, Olmedo $\mathrm{M}$, et al. Oxigenoterapia de alto flujo con cánula nasal en pacientes con insuficiencia respiratoria aguda. Rev Clin Esp. 2014;214(Espec Congr):975. doi: 10.1016/j.arbres.2015.03.015.

36. Lee MK, Choi J, Park B, et al. High flow nasal cannulae oxygen therapy in acute-moderate hypercapnic respiratory failure. Clin Respir J. 2018;12:2046-2056. https://doi.org/10.1111/crj.12772.

37. Schwabbauer N, Berg B, Blumenstock G, Haap M, Hetzel J, Riessen R. Nasal high-flow oxygen therapy in patients with hypoxic respiratory failure: effect on functional and subjective respiratory parameters compared to conventional oxygen therapy and non-invasive ventilation (NIV). BMC Anesthesiol. 2014;14:66. Published 2014 Aug 7. doi: 10.1186/1471-2253-14-66. 